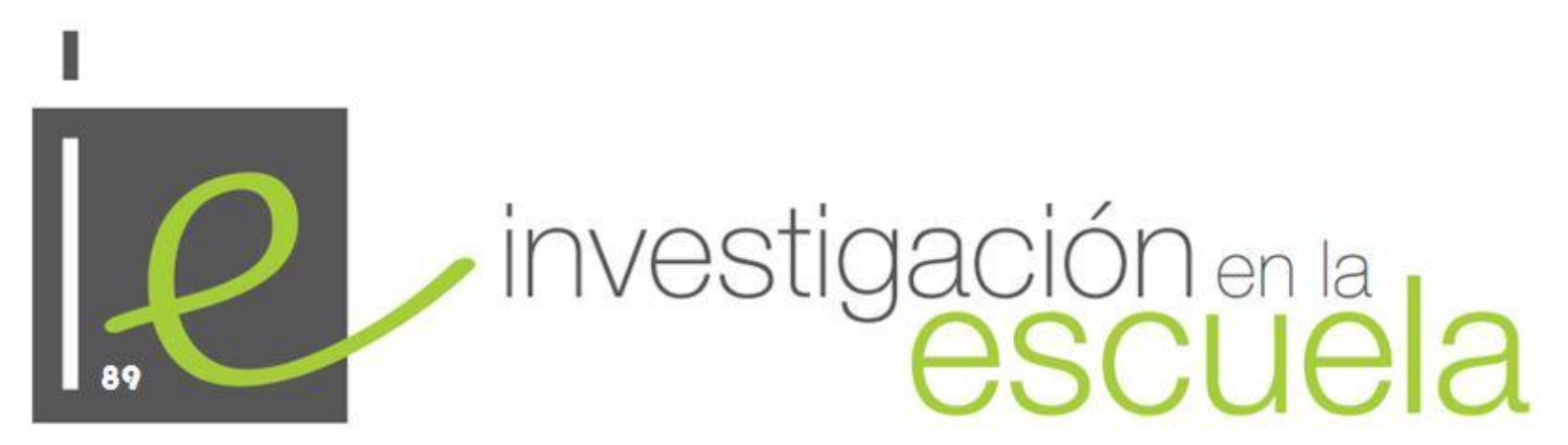

Revista académica evaluada por pares y de acceso abierto

\title{
El diálogo en el aula y la elaboración compartida de historias narradas.
}

\author{
Belén Irquierdo-Magaldi \\ Universidad de Cantabria \\ España
}

Citación: Izquierdo, B. (2016). El diálogo en el aula y la elaboración compartida de historias narradas. Investigación en la Escuela, 89, 17-34. Recuperado de:

http://www.investigacionenlaescuela.es/articulos/R89/R89-2.pdf

Resumen: Nuestro propósito ha sido explorar una práctica de escritura en un contexto colaborativo, en la que docente y estudiantes de 14 años trabajan juntos en actividades planificadas como talleres, dialogando, y compartiendo reflexiones que les permiten comprender y transformar los significados de la realidad que construyen, y de esta manera, progresar en su competencia escrita. La interacción comunicativa se observa en pequeños grupos de trabajo, diferenciando cuando el grupo lo componen los iguales, y cuando en el grupo participa la docente como un miembro más. Los resultados del análisis discursivo muestran la importancia del rol de guía que en las diferentes fases de composición escrita ejerce la profesora. De manera singular, el grupo de iguales es capaz de asumir el control de la situación de escritura, tanto en la organización de las relaciones sociales, como en la estructuración de la tarea.

Palabras clave: "Planificación textual"; "discurso educativo"; "estrategias discursivas"; "grupos colaborativos”.

Título en inglés: Dialog in the classroom and shared elaboration of narrated stories. Abstract: Our purpose has been to explore a writing practice in a collaborative context, in which teacher and students of 14 years old work together in planned activities such as workshops, 
dialoguing and sharing reflections which allow them to understand and to transform the meanings of reality that they build, and in this way, to progress in their writing skills.

The communicative interaction is observed in small working groups, differentiating when the group is made-up by equals, and when the teacher participates as a member of the group. The results of the discursive analysis show the importance of the guiding role within the different phases of the writing composition performed by the teacher. In a singular manner, the group of equals is able to assume the control over the writing situation, in the organization of social relationships as well as in the structuring of the task.

Key words: "Textual planning"; "educative discourse"; "discursive strategies"; "collaborative groups".

Título en francés: Le dialogue dans la classe et l'élaboration partagée des histoires narrées. Résumé: Notre but c'a été d'explorer une pratique d'écriture dans un contexte de collaboration où l'enseignant et les étudiants à 14 ans travaillent ensemble sur des activités planifiées telles que des ateliers, ils dialoguent, et partagent des réflexions qui leur permettent de comprendre et de transformer les signifiances de la réalité qu'ils construisent, ainsi, progresser sur sa compétence écrite. L'interaction communicative on l'observe dans des petits groupes de travail, on différence quand le groupe est composé par des égales, et quand dans le groupe participe l'enseignante comme un autre membre. Les résultats de l'analyse discursive montrent l'importance du rôle de guide que dans les différentes phases de la composition écrite exerce l'enseignante. Singulièrement, le groupe d'égales est capable d'assumer le control de la situation écriture, á la fois dans l'organisation des relations sociales et dans l'structuration de la tâche.

Mosts clé: "Planification textuelle"; "discours éducatif” ; "stratégies discursives"; "groupes collaboratives".

\section{Metas escolares y exigencias sociales}

La transformación de las condiciones sociales y tecnológicas en las que vivimos requiere personas competentes que demuestren su capacidad de adaptación a multitud de contextos educativos, sociales y laborales a lo largo de su vida. Sin embargo, los resultados relacionados con las competencias en lectura y escritura de nuestros estudiantes en la última década, no han sido favorables para nuestro sistema educativo. Esto nos hace pensar en la distancia que existe entre las metas escolares y las exigencias sociales. Tales discrepancias reiteran la necesidad de cambio en el papel de los agentes educativos. Pensamos que la labor central del docente es ayudar a los estudiantes a progresar en su competencia letrada, a través de procesos de enseñanza y aprendizaje en los que se tome conciencia de las características y utilidad que nuestra cultura atribuye a los distintos estilos textuales.

Por esta razón, nos acercaremos desde el ámbito educativo en el que nos movemos, a investigaciones enmarcadas en enfoques socioculturales (Hammersley y Atkinson, 2007; Spindler y Hammond, 2006) y sociolingüísticos (Bakhtin, 1981; Barton y Papen, 2010; van Dijk, 2011; Gee, 2008) que han orientado el estudio de las actividades comunicativas y de aprendizaje de la composición escrita, además de considerar que la conversación mantiene vivo el propio lenguaje al utilizarlo como instrumento comunicativo en situaciones de interacción (Mercer y Hodgkinson, 2008).

Respecto a los procesos de composición escrita, contamos con antecedentes de estudios dedicados a la escritura como proceso de resolución de problemas y los que lo entienden como un 
proceso de naturaleza social que permite interpretar el mundo. En este sentido, el proceso de alfabetización implica desde su inicio la adquisición de conocimiento con conciencia de su utilidad y función social, como vía de construcción de futuros escritores, en la línea de los modelos constructivistas (Ferreiro, Teberosky y Castorina, 2004) que nacen de las prácticas educativas para transformarlas. Hablamos del desarrollo de una competencia relacionada con "trasformar el conocimiento", en la línea clásica de (Bereiter y Scardamalia, 1987) que en el ámbito educativo actual se entiende como reflexión metacognitiva y se considera básica en el proceso de elaboración escrita (Álvarez Angulo, 2006; Camps y Uribe, 2008) a través de una práctica sistemática de enseñanza y aprendizaje desde la escuela hasta la universidad.

La búsqueda de nuevos contextos educativos adaptados a los cambios sociales de los últimos años, ha orientado nuestra apuesta por el diseño de un contexto escolar que promueva y facilite el desarrollo de habilidades relacionadas con la alfabetización en el medio impreso en el que sus participantes se sientan implicados. En trabajos anteriores (Lacasa y GIPI, 2006; Lacasa y Reina, 2004), se comentan ampliamente propuestas de este tipo, en el que investigadores, docentes y estudiantes convierten el aula en talleres abiertos a contenidos y participantes más allá del curriculum escolar.

\section{Metodología}

Nos hemos acercado a la Etnografía de la Comunicación porque nos permite fundamentar teóricamente nuestra investigación y a la vez nos proporciona elementos metodológicos que se pueden adaptar a la investigación de la actividad educativa como fenómeno social y comunicativo (Gee, 1999), en la que los aspectos comunicativos, cognitivos y sociales se integran como un proceso entrelazado al contexto en que se desarrolla (Hammersley y Atkinson, 2007).

La observación participante forma parte de la metodología que se puede emplear en este tipo de investigaciones cuando interesa observar desde dentro y reflexionar sobre las prácticas educativas (Spindler y Hammond, 2006) que se llevan a cabo habitualmente o en las que se quiere introducir alguna experiencia innovadora. En nuestro estudio, un equipo interdisciplinar compuesto por docente e investigadores colabora en la planificación y diseño de una actividad educativa en un contexto escolar innovador.

El punto de encuentro de la perspectiva etnográfica con la Psicología Sociocultural nos ayuda a entender la importancia de la interacción en los procesos de enseñanza y aprendizaje. En nuestro estudio partimos de una noción vygotskyana de Zona de Desarrollo Próximo que no se agota en la relación interpersonal experto-novato sino que se extiende al grupo participante, como una comunidad de aprendizaje que tomando distintos roles, crea activamente un contexto sociocultural propio, situado (Rogoff, 1994 y 2012), para definir el contexto colaborativo de trabajo de nuestro taller de escritura. En estos entornos educativos, el profesor se responsabiliza de las prácticas comunicativas, las actividades sociales conjuntas y el progreso intelectual de alumnos y alumnas que trabajan en grupos conjuntamente (Mercer y Hodgkinson, 2008). El análisis de la conversación que mantienen docentes y estudiantes nos acercará a las estrategias que utilizan en su construcción de significados compartidos.

\section{El taller de escritura en un contexto colaborativo}

Los objetivos específicos de investigación negociados con el equipo docente se enumeran a continuación: 
1. Planificar un contexto escolar colaborativo de composición narrativa a partir del currículum escolar de educación secundaria.

2. Describir los procesos de participación guiada que tienen lugar cuando se interactúa en pequeño grupo en tareas de escritura narrativa con una meta común.

3. Analizar cómo evoluciona el proceso de enseñanza-aprendizaje relacionado con prácticas alfabetizadoras escolares de construcción de historias.

4. Analizar dos situaciones de trabajo en pequeño grupo por separado:

a. La presencia en el grupo de la docente.

b. La interacción con los iguales.

\section{Participantes, diseño y registro de los datos}

En este caso, un centro educativo de nivel socioeconómico medio-bajo, dependiente de la Consejería de Educación de la Junta de Andalucía, en el que se implementaron, los talleres de escritura entendidos como espacios en los que se promueve la integración de recursos de la vida cotidiana (cultural y social) con los contenidos escolares (Izquierdo-Magaldi, 2011).

Una actividad extraescolar, planificada con anterioridad al taller de escritura, se eligió para acercar e integrar los contenidos tradicionales del currículum con experiencias reales del alumnado. Para la elaboración de textos narrativos en el aula, se planificaron un total de tres sesiones a lo largo de tres semanas dentro del cronograma escolar. Cada sesión semanal era de 90 minutos de duración. El grupo con el que trabajamos, del que la profesora era responsable, estaba compuesto por un total de 17 alumnos y 16 alumnas de un nivel educativo equivalente a $2^{\circ}$ de Educación Secundaria Obligatoria. El espacio se dividió en mesas de trabajo para pequeños grupos de 6 componentes que se mantuvieron durante toda la actividad. Los alumnos y alumnas que formaban parte del grupo $1 \mathrm{y}$ 2 (G1 y G2), fueron los elegidos para poder registrar de forma simultánea su trabajo y poder comparar con los mismos estudiantes, su trabajo en presencia de la profesora o en el grupo de iguales, tal y como se plantea en los objetivos del estudio.

Los datos registrados y digitalizados correspondientes a las grabaciones de las tres sesiones narrativas suponen 270 minutos de material de vídeo y 270 minutos de material de audio en total. Ofrecemos seguidamente un esquema simple (figura 1) de los recursos empleados.

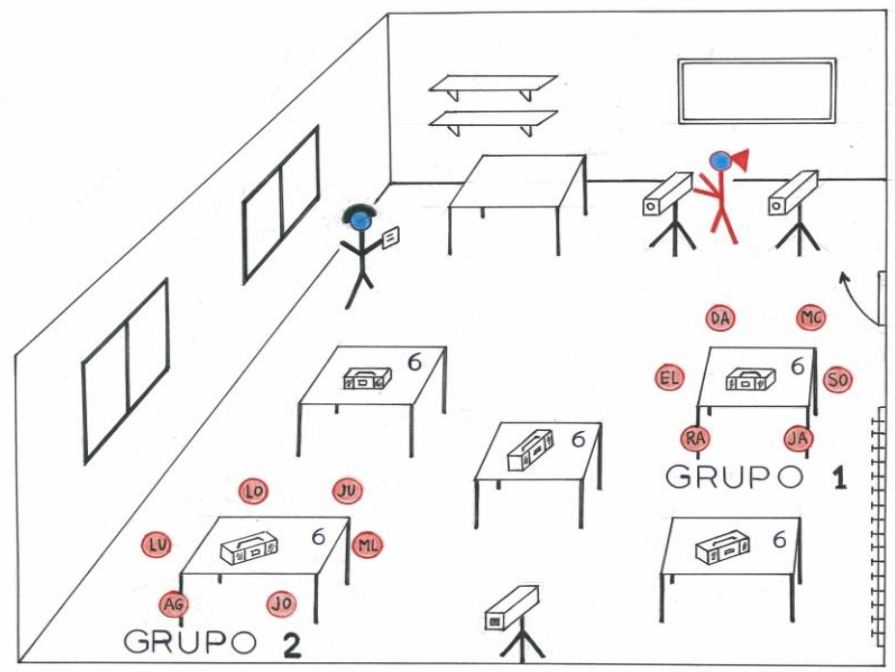

Figura 1. Croquis de la organización y registro de vídeo y audio en el aula. 


\section{Interpretación de los datos}

La posibilidad de realizar una interpretación cualitativa y cuantitativa complementarias ofrecen una visión más completa de la realidad, necesaria para otorgar validez al estudio (Denzin, 2010; Dörney, 2007). Por una parte, se analiza la organización educativa centrada en la planificación de la actividad y los contenidos tratados. Por otra parte, la organización social de la actividad en pequeños grupos colaborativos, atendiendo a la interacción comunicativa en la que se apoya el progreso en el aprendizaje. Como señalan Coll y Onrubia (1996) al hablar de la unidad de análisis hay que dar cuenta de lo micro, refiriéndose a los dispositivos y recursos semióticos empleados por los participantes; y de lo macro que coincide con las formas de organización de la actividad conjunta. La articulación de ambos planos de análisis se consigue al tener en cuenta la dimensión temporal (Hühn, 2009) en la que se suceden las sesiones y que representa el hilo conductor de la actividad global. Es decir, dentro de cada sesión existe una secuencia temporal guiada por la docente y distribuida en diferentes momentos o fases de trabajo que se repiten como una rutina de elaboración escrita para cada día (lo macro). A la vez, los turnos del diálogo entre docente y estudiantes se toman como unidades comunicativas de participación, inseparables a su vez de la información precedente y posterior, que se representa en unidades significativas más amplias como el mensaje. De forma inductiva, a partir de los documentos transcritos hemos examinado y clasificado las pautas seguidas en el diálogo del aula, para finalmente construir una descripción de las estrategias discursivas empleadas (lo micro).

\section{Dialogar, escribir y editar en el aula}

En este apartado narramos paso a paso cómo docente e investigadores negocian la introducción de algunas propuestas de interés para los participantes del taller (objetivo 1), teniendo presente que en la composición escrita no se pueden desligar el proceso de elaboración y la funcionalidad del mismo.

\section{El aula como lugar de encuentros significativos}

El punto de partida del taller se planificó con anterioridad al trabajo de aula, la profesora organiza una actividad extraescolar que se pueda enlazar con los contenidos programados alrededor de la obra del experto escritor por excelencia, Cervantes. Todos los estudiantes tienen la oportunidad de asistir a una exposición y representación teatral de Don Quijote de la Mancha en el Museo Diocesano de Córdoba. La experiencia compartida sirvió de información previa para elegir el contenido de las sesiones del taller de escritura.

Ya en el aula, en la primera sesión (S1), la profesora introduce la actividad global del taller de escritura y expone un doble objetivo, por una parte comunicar la finalidad del taller, "escribir textos interesantes para que los puedan escuchar y leer al final de cada sesión, los compañeros Y por otra parte, introducir la información necesaria para que todos los participantes puedan partir de un contexto de trabajo compartido, “....Entonces para...preparar ahora algún trabajito, para que hagamos algo de expresión escrita, pues claro, buscamos algo también que os atrajese...". Cuando se refiere a "buscamos", incluye al equipo de investigación y la planificación previa la actividad. Al mismo tiempo, recuerda junto con sus estudiantes la finalidad de la actividad extraescolar y su relación con el taller de escritura: "siempre pensamos...que íbamos a trabajar algo con Don Quijote, pero vaya, de una manera divertida, no para volver a leernos el Quijote, ni nada de eso... para que recordemos un poco cómo es Don Quijote y cómo es Sancho Panza, ¿uhm? Como allí la vivisteis, 
después aquella máquina tan bonita ¿no? de los molinos que os llamó muchísimo la atención...”. Las anotaciones de la profesora servirán para seleccionar los temas o contenidos de las tareas de escritura de cada una de las sesiones: "os dije que...que...me pusierais en un papel la experiencia...la experiencia que...que habíais sentido cuando visteis la representación aquella. Y claro, me he leído todas las contestaciones vuestras y entonces saqué, bueno pues como algunas notas".

Más allá del espacio físico que supone el aula, docente y estudiantes con una meta común, se involucran en un proceso mental de contextualización (Mercer, 2001) cuando enlazan y construyen de forma dinámica en cada encuentro los contenidos que forman parte del discurso escolar. En la figura 2, se recogen los contenidos que sirven para conectar la experiencia compartida fuera del aula con la tarea de escritura y que se negociaban al inicio de cada sesión.

En la primera sesión narrativa (S1), el contenido de la composición escrita gira alrededor del personaje del escudero que Don Quijote tendrá que buscar trasladándose en el tiempo a la actualidad. En la segunda sesión (S2), los estudiantes cambiarán de escenario a los protagonistas para escribir nuevas aventuras. En la tercera sesión (S3) con la que finaliza el taller, el contenido versará sobre la recuperación de la razón por parte de Don Quijote.

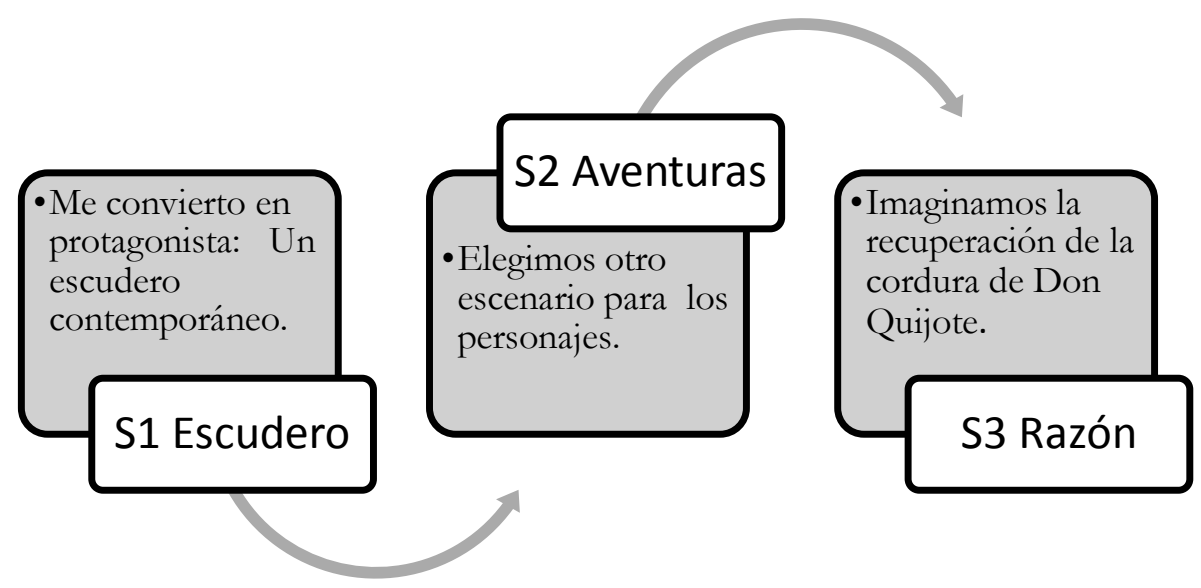

Figura 2. Contenidos de las historias elegidos para cada sesión.

\section{Construcción narrativa conjunta}

La docente establece un diálogo con los estudiantes en el que trata de transmitir el aspecto pragmático de la composición escrita necesario para establecer una conciencia del mismo, un verdadero proceso sociocognitivo (objetivo 2 y 3 ).

La secuencia temporal de trabajo seguida en cada sesión, comienza con el trabajo en gran grupo en el que la docente introduce la finalidad del taller y se comparte con toda la clase. A continuación, la clase se organiza en pequeños grupos para planificar su narración elaborando un esquema común de trabajo. Una vez finalizado el esquema, cada miembro del grupo escribe su interpretación, es la fase de textualización. La secuencia finaliza con la reorganización de los alumnos de nuevo en gran grupo para analizar todo el proceso y su producto cuando leen el texto elaborado ante los compañeros y compañeras, como evaluación-revisión al término de cada sesión. A continuación, en la figura 3, se detalla el esquema seguido en el que las flechas en sentido circular reflejan el feedback que se establece en todo el proceso. 


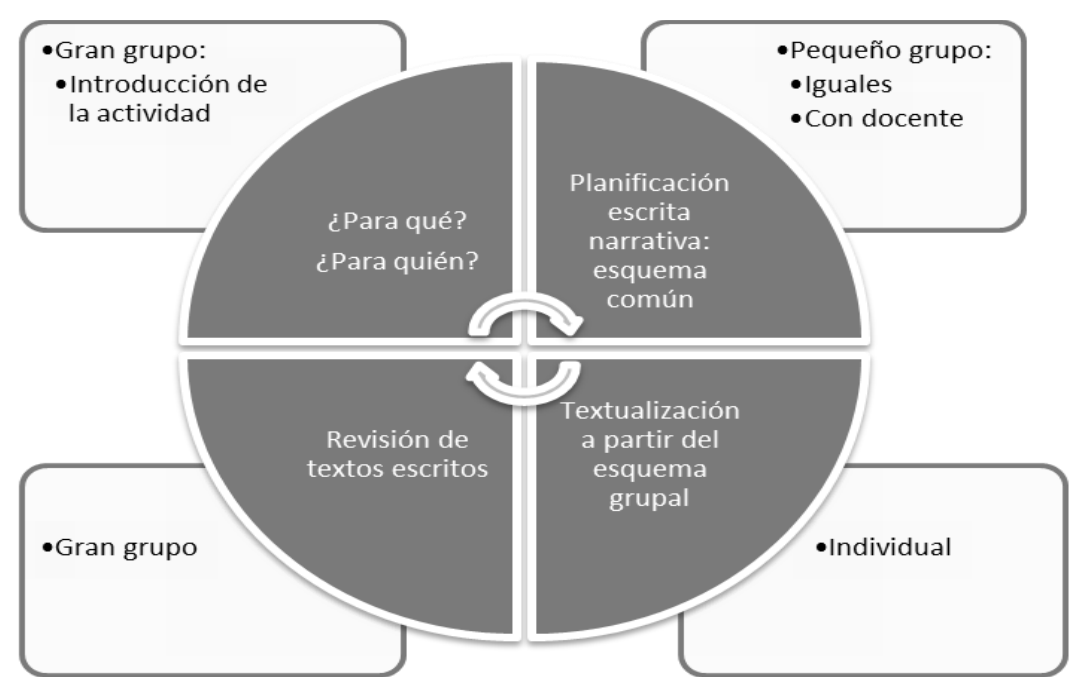

Figura 3. Proceso sociocognitivo de composición escrita en cada sesión.

En los siguientes apartados trataremos de explicar con ejemplos concretos todo el proceso referido.

“Cómo lo vamos a hacer”. Planificación, textualización y revisión. En el taller, el papel de enseñantes como líderes lo pueden ejercer tanto la profesora como los compañeros. Hablamos de una participación guiada (Rogoff, 2008) que inicialmente es dirigida por la docente pero con posibilidad de traspasar el control al grupo de iguales.

El trabajo colaborativo en pequeños grupos propicia la implicación en la elaboración de historias cuya utilidad comunicativa la docente anticipa y repite a lo largo de las sesiones. En la primera sesión (S1), a lo largo de los diez minutos de su intervención, especifica el procedimiento. Mostramos el discurso de la docente en el que hemos eliminado las partes del mismo (señaladas con el símbolo "//") que no aportan información en este caso: “¿Cómo lo vamos a hacer? Mirad, en primer lugar, lo que vais a sacar unas notas común en el grupo ¿eh?, os vais a poner de acuerdo // teniéndolo como modelo.// O sea que ponéis en común ¿eh?, en común // Uno es la secretaria o secretario, como queráis, ¿ंeh?// con esas ideas que tenéis en común, empezáis a desarrollar el texto y cada uno el suyo, cada uno el suyo. //Pero atendiendo también al grupo".

En el texto manuscrito, figura 4, se puede comprobar el desarrollo del proceso en el que en grupo planifica la “invención de una aventura en la que Don Quijote recupera la razón”. Volcar el pensamiento sobre el papel requiere un esfuerzo personal fruto de un trabajo situado podríamos decir. El proceso de composición escrita planificado en una situación comunicativa en la que se han logrado hacer explícitas las finalidades que sus participantes han negociado y compartido deja huella en el discurso escrito. La base del proceso de elaboración escrita descansa en la reflexión metacognitiva como vemos en la realización del esquema común (rodeado por la elipse). Primero han aportado ideas sobre el contenido de la historia que han ido escribiendo consecutivamente. Posteriormente han organizando la secuencia narrativa numéricamente, del 1 al 5, utilizando un criterio de orden temporal. El último paso consiste en personalizar la historia y buscar un título representativo como en este ejemplo, "Don Quijote se pone cuerdo". El desarrollo de la historia sigue el orden establecido por el grupo en el primer párrafo, para continuar con soltura la tragicomedia que acaba con un final feliz, plenamente identificado con el entorno actual del escritor que ha reinventado la aventura de Don Quijote. 


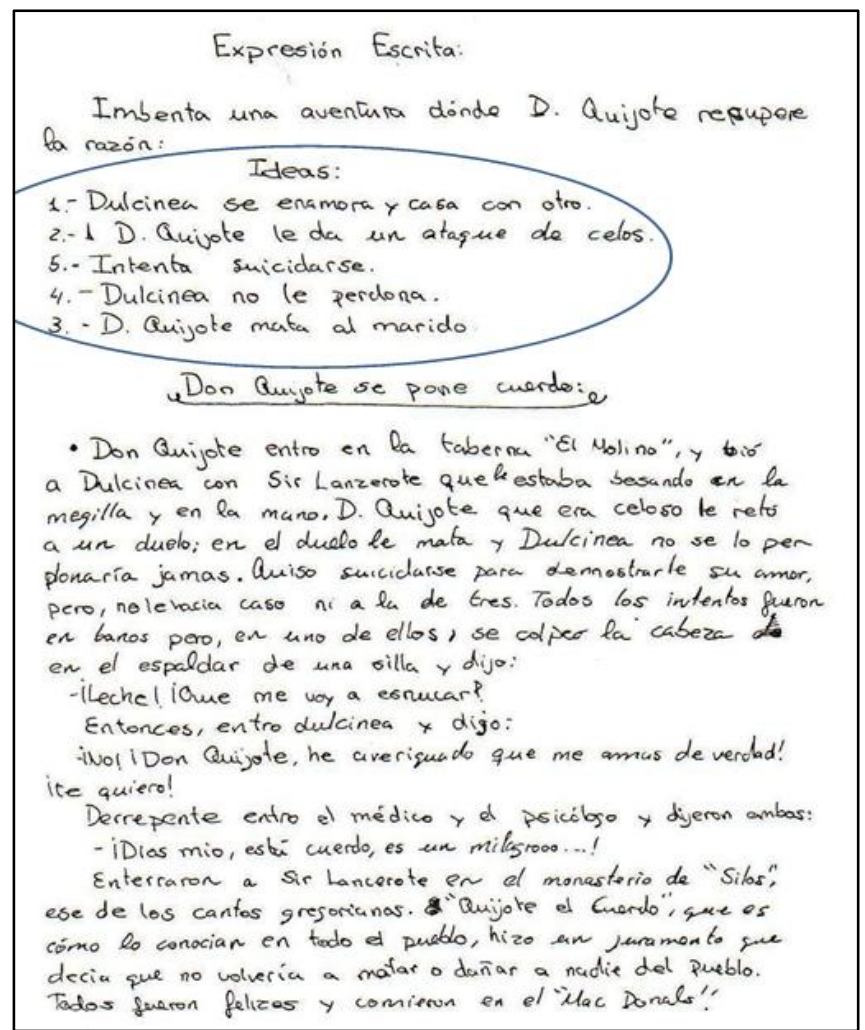

Figura 4. Proceso sociocognitivo de composición escrita reflejado en el producto individual

La composición escrita comienza con la preparación de la tarea, continúa con la escritura organizada del texto y finaliza con la revisión del proceso y el producto final como pilares de una progresión cognitiva individual en un proceso aparentemente lineal. Sin embargo, podemos hablar de etapas conectadas entre sí que permiten progresar en ambas direcciones durante todo el proceso a través de la reformulación de la estructura y del contenido de composición escrita (Camps y Uribe, 2008), por lo que la composición escrita en el taller se transforma en un proceso sociocognitivo. Es decir, el progreso individual se enriquece con el trabajo en grupo cuando se construye una versión de la realidad narrada en colaboración.

\section{Evolución del taller en el tiempo. Estrategias discursivas}

Hablamos de estrategias sociocognitivas entendidas como recursos de control y seguimiento en la construcción conjunta de significados (Cubero, Cubero y Santamaría, 2008; Saló, 2006) y suponen una agrupación descriptiva de los patrones que se repiten y su función, en la evolución del diálogo del taller. En la figura 5 mostramos de forma esquemática las cuatro estrategias que hemos definido, a partir del análisis de los datos transcritos y codificados. 


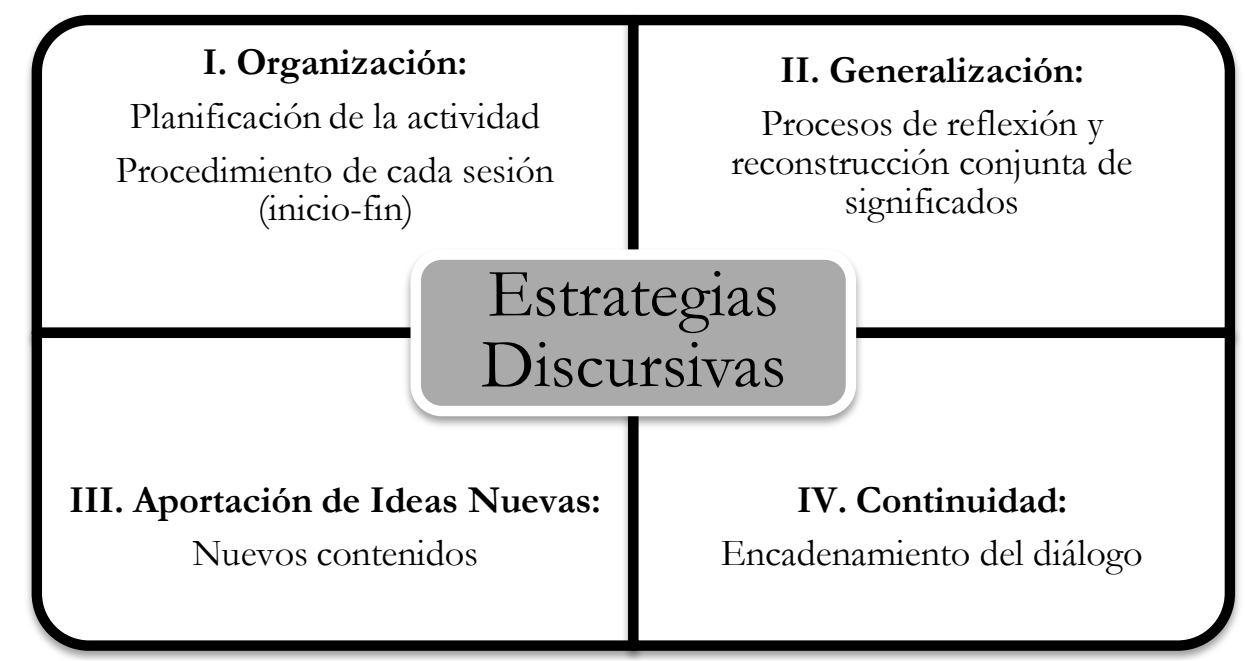

Figura 5. Estrategias discursivas en el taller de escritura

Expondremos brevemente la función de las estrategias discursivas I y II y aportaremos fragmentos de conversación de docente y estudiantes relacionadas con la organización y generalización de significados por ser las estrategias definidas con mayor peso en la construcción de historias (frecuencia de aparición), ya que las estrategias III y IV de aportación de ideas nuevas y las estrategias de continuidad han supuesto un apoyo al encadenamiento de los turnos de conversación.

\section{Estrategias de Organización}

Distinguimos dentro de las estrategias de organización, unas de carácter más amplio como son las estrategias de organización global que introducen la actividad, anticipan información sobre la actividad y sus fases de ejecución y desarrollo. Consisten en referencias explicitas al discurso, o a las tareas precedentes ("Información dada"). Sirven para crear el contexto sobre el que se construye el diálogo.

Otras más específicas o puntuales como las estrategias de organización concreta, instrucciones y pautas precisas que informan del procedimiento de cada tarea. Se relacionan con el mantenimiento de la interacción en el aula, en el que están presentes de manera implícita las rutinas escolares como escuchar, hablar ordenadamente, etc.

Por último, las estrategias de conclusión indican el término de las tareas que corresponden a cada sesión de la actividad general. Se evalúa ante toda la clase el trabajo realizado, a través de los comentarios de la docente.

Fragmento de conversación I.a: La profesora actúa de guía en la organización de la primera a la última sesión. La profesora verbaliza explícitamente el procedimiento más adecuado para elaborar la última de las historias del taller en el siguiente fragmento de conversación. 


\title{
Personajes y otros criterios S3 G2 Narración: Razón
}

\begin{abstract}
Profesora: Hoy vamos a hacer el último texto que va a ser narrativo. Vamos a contar una aventura ceh?, que ya hemos explicado el amor de D.Q. que os había llamado la atención. La locura también. Y luego las aventuras de D.Q. también os gustaba. Por lo menos es lo que vosotros habiais dicho, lo que os había llamado la atención. Entonces hoy vamos a hacer ya, lo último céh? que va a ser un texto narrativo también...y vamos a...contar una aventura (...) (mirando sus papeles). Lo tengo aquí. iAh, ya!. Una aventura...en la que D.Q. recupere la razón. Ya lo dejamos cuerdo y ya <nos despedimos de ék. ¿Vale? (...). Entonces lo voy a poner ahí en la pizarra. ¿? Inventa, ¿eh? inventa...o cuenta una aventura en la que D.Q.... Doy la orden y la voy a poner en la pizarra. Entonces cqué tendremos que hacer? Pues lo de siempre eno? los personajes ya Tos tenemos...ंeh? Tendremos que pensar en qué tipo de aventura.... Cómo se va a desarrollar esa aventura, los puntos más importantes de esa aventura... ¿eh? en grupo...ordenar esos puntos y luego ya cada uno hace su creación y le pone un título. Pero... ¿eh? hoy ya D.Q. va a recobrar la razón. Y cuando ya lo dejemos con su razón recobrada...ya... ¿eh?...descansaremos de él....
\end{abstract}

Figura 6. Intervención de la profesora como guía de la actividad educativa

De la primera sesión a la última, la profesora repasa las instrucciones relacionadas con el procedimiento ligado a la tipología textual narrativa que consiste en hacer "lo de siempre", tener en cuenta los elementos esenciales como los personajes, el tipo de aventura y los puntos más importantes que se quieren contar de la aventura. Indica que la tarea es grupal, tanto la búsqueda del contenido como la tarea de ordenar las ideas. Se trata de la fase de planificación textual en la que los estudiantes elaboran un esquema común de trabajo. La profesora adelanta lo que corresponde a la fase de trabajo individual, "cada uno hace su creación", con lo que remarca la responsabilidad individual en la composición escrita. La última instrucción, "y le pone un título" al texto individual intensifica de nuevo el carácter individual del proceso de escritura. Pero no olvidemos que ese producto escrito llevará la huella de la planificación realizada en grupo, base fundamental para la interpretación y construcción individual.

Fragmento de conversación I.b: Cuando el grupo de iguales estructura la historia. Comprobamos con el siguiente ejemplo que el grupo de iguales es capaz de controlar las rutinas escolares empleadas por la profesora, entre todos hacen que funcione el grupo, gestionando y activando la participación conjunta de forma eficaz. 


\section{Qué clase de aventura S2 G2 Narración: Aventuras}

1. $M^{a} L .:$ ¿Os habéis enterao? porque yo no me he enterao de na.

2. Luci: pon una aventura ¿?.

3. Lore: sí.

4. $\quad M^{a} L$.: otra vez una bolsa de aventuras, ijoer!

5. Luci: pon...unas cataratas.

6. Lore: entonces ¿? la aventura y los puntos más impor/ (habla a la vez Lucía).

7. Luci: en cataratas... cataratas.

8. Jose: ¿? ¿Qué quieres que se ponga aquí encima? ¿? ¿encima?

9. Luci: Pon unas cataratas....unas cataratas (...). Un lugar, un tiempo.

10. Jose: ¿? a ver si...

11. Luci: ¿qué hay que poner un lugar dónde....? (se dirige a $M^{a} L$.)

12. $M^{a} L$.: un título (...) un título. ( $M^{a} L$. estaba mirando hacia atrás y al volverse sigue sin tener en cuenta lo que dice Lucía. Mira a Jose pidiendo un título).

13. Luci: "D. Q. en las cataratas". (riéndose, se miran ella y $M^{a} L$., parece que no sabe si tomárselo en serio o en broma).

14. Jose: ¿Pero qué hay que hacer?

15. Lore: Pero primero tendremos que empezar la aventura para ponerle un título.

16. Agus.: iclaro!

17. $M^{a} L .:$ clase de aventura (sigue marcando criterios propios).

Figura 7. El grupo de iguales planificando aventuras

En el turno 2 y 5 Luci organiza el trabajo del grupo asociando la narración de una aventura con un lugar especial "las cataratas". En el turno 6 su compañera Lore sigue las instrucciones que ha dado la profesora al inicio de la sesión para encuadrar el trabajo, refiriéndose a la clase de aventura y puntos más importantes (estructura narrativa). Hasta el turno 13 Luci no ceja en su empeño por situar la aventura en unas cataratas, utilizando criterios de lugar y tiempo (turno 9). A su vez, $\mathrm{M}^{\mathrm{a}}$ Luisa intenta otro camino pidiendo el título (turno 12) al resto del grupo. De nuevo es Lore quien suaviza la división del grupo centrando el procedimiento a seguir en el turno 15 y Ma L. toma las riendas utilizando el criterio "clase de aventura" (turno 17), para organizar la entrada de contenidos previos al título.

\section{Estrategias de Generalización}

Su función básica es el intercambio de conocimientos en contextos de actividad conjunta, alrededor de unos contenidos. Se emplean preguntas con respuestas si-no, categoriales (cómo, qué, cuándo, dónde, por qué?...) y metacognitivas (qué quiere decir), con las que se van introduciendo elementos del tema tratado. El contenido o significado se consigue a través de las contribuciones de la docente y de los estudiantes en una reflexión compartida

Fragmento de conversación II.a: Cuando la profesora favorece el pensamiento a través del diálogo narrativo. En el ejemplo siguiente comprobamos que desde el mismo momento que la profesora empieza a trabajar con el grupo intenta establecer el contexto compartido para progresar en la reflexión conjunta sobre el contenido que manejan (turno 1). Se parte como en el resto de las sesiones de la lectura de un pasaje del Quijote, en esta sesión, de lo acontecido en la venta donde Don Quijote se convierte en caballero. La profesora se refiere a la información dada previamente para favorecer que el grupo introduzca nuevos contenidos pero no al azar. Anima a través de sus preguntas (turnos 12 y 14) a reflexionar sobre la adecuación de los elementos narrativos como el 
título, al contenido de la historia que van a elaborar ("vamos a ver, cómo puede ser el escudero", "¿un nombre cualquiera le ponemos al escudero?"), refiriéndose a las características que identifican al personaje.

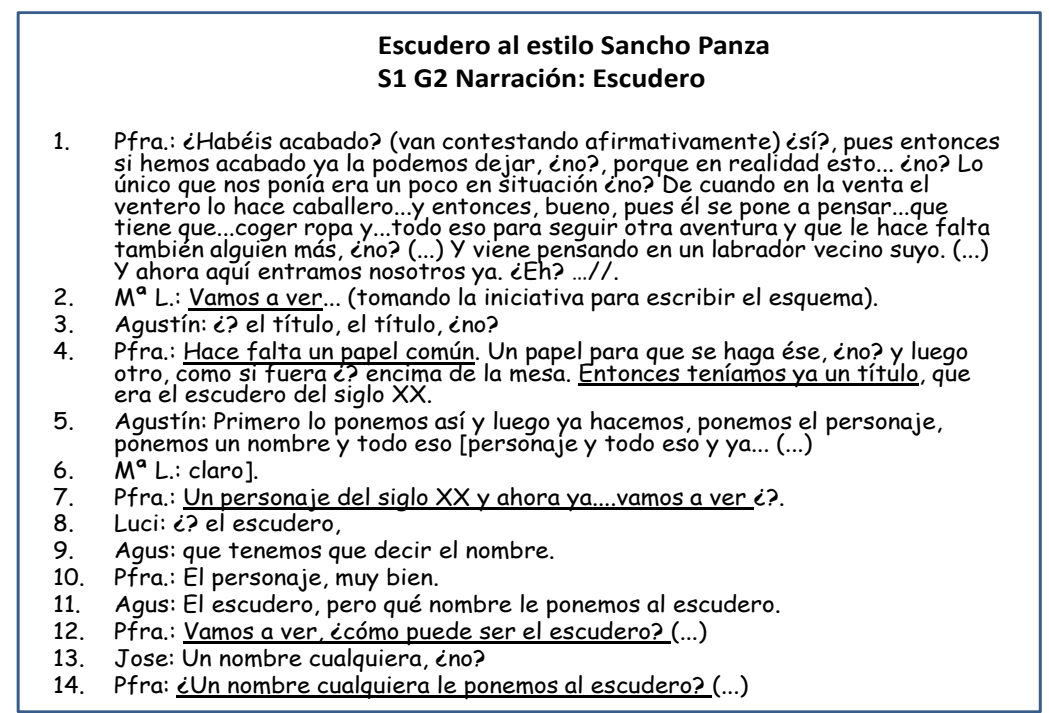

Figura 8. Fragmento de conversación: profesora e iguales reflexionando sobre el protagonista

Podemos decir que la reflexión que suscita el diálogo ante la composición narrativa se centra en el análisis de su estructura, concretamente en el peso de los protagonistas y en la acción, para promover una interpretación conjunta que lleve a la creación de una historia propia.

Fragmento de conversación II.b: Cuando los iguales piensan conjuntamente. Con otro ejemplo trataremos de reflejar cómo el grupo de iguales también es capaz de reflexionar sobre el contenido y características de elementos narrativos que han elegido para su historia.

\section{Qué contamos \\ S1 G1 Narración: Escudero}

1. M. C.: Tenemos que sacar ideas y luego ya cada uno hace su descripción y lo ordena.

2. Sora: Pues venga, vamos a hacerlo.

3. Dani: Ideas, Dios mío, ideas ¿? Dios mío, tengo miedo. Saca las ideas, Dios mío.

4. Javi: Las ideas....... Don Quijote..... contra los charlies...

5. Sora: ¿? Don quijote.

6. Javi: ...C?... Don Quijote por Vietnam?

7. M.C.: No.

8. Javi: Era.... erase una vez un loco chiflado que...llamado Don Quijote...No, mira,

podemos poner: erase una vez.....un hombre tan chiflado que empezo a leer el

mina en la cabeza..... ipshshsh!....y de repente creía que era Don Quijote.

9. M. C.: No, pero es que Don Quijote, de verdad, tiene que ser Don Quijote de...en verdad....Luego ya un personaje que nosotros pongamos.

10. Dani: Venga, vamos a pasar de historia. Ya tenemos esto... hemos buscado las ideas...es que, es que ya....que nosotros nada más que sacamos las ideas y luego cada uno empieza a hacer su historia.

11. Sora: Trae, que me lo des.

12. J. J.: Hay que sacar las ideas y después...

13. Sora: Y después...no, no. Nosot/...hemos sacado una idea, lo que pasa es que Sora: Ýs después...no, no. Nosot/...hemos sacado una

14. Javi: Un momento. Estando Don Quijote por la selva del Vietnam tuvo la idea de buscar un escudero, entonces los ruidos de un bazzooka le sorprendieron (rie).

Figura 9. Fragmento de conversación: el grupo de iguales reflexionando sobre su historia 
Intentan seguir el procedimiento de planificación que se repite en todas las sesiones con la elaboración de un esquema común en el que el grupo escribe las ideas que han aportado y negociado para narrar su historia (turno 1). En la búsqueda del contenido, algún miembro del grupo (turno 9) se da cuenta de que no están cumpliendo con la tarea y realiza una pequeña reflexión sobre el protagonista de la historia, tiene que ser "de verdad", lo que orienta de nuevo al grupo para reconstruir la historia de partida alrededor de un nuevo personaje, un escudero contemporáneo (turno 14).

Comentarios, reflexiones, generalizaciones y transformaciones forman parte del intercambio de conocimientos, en los que la figura de la docente o algún miembro del grupo encamina la interpretación que se está elaborando hacia una comprensión compartida (Mercer, 2001) dentro del proceso de participación guiada que amplía la comprensión individual de los participantes (Rogoff, 2008). Las historias se convierten de esta manera en objetos de reflexión consciente, característica que convierte a la escritura en vehículo esencial en el aprendizaje.

\section{Evolución de los grupos de trabajo en el tiempo}

Hemos seleccionado los datos numéricos expresados en porcentajes y representados en las figuras que aportamos en este apartado para entender la presencia y evolución de los mecanismos interactivos a lo largo de las tres sesiones narrativas tanto en el trabajo de grupo con presencia de la profesora, como en el trabajo del grupo de iguales (objetivo 4).

En la figura "Interacción con docente Narraciones", interpretamos cómo la relación asimétrica que establece la docente cuando trabaja con toda la clase se diluye al unirse al grupo. Las estrategias utilizadas a lo largo de las sesiones, oscila entre un $12 \%$ y un $2 \%$, en este sentido podemos decir que el papel de la profesora en el progreso sociocognitivo como guía tiene que ver en el mantenimiento de las rutinas de trabajo a lo largo la actividad educativa. Más que dirigir, intenta ceder protagonismo al resto de los miembros, dosificando la ayuda necesaria. En el trabajo colaborativo en grupo los papeles de sus integrantes se ajustan a las exigencias de la tarea en cada momento, ejerciendo de líder uno o varios de los participantes para organizar o reflexionar sobre las tareas de escritura, sin que se de un control total ni centralizado en una sola persona.

Resaltamos el papel de la profesora al final de cada sesión cuando concluye con la revisión de textos a través de la lectura individual ante la audiencia de la clase. La evaluación final sirve de feedback del trabajo realizado y de contexto mental compartido de sesión en sesión. 


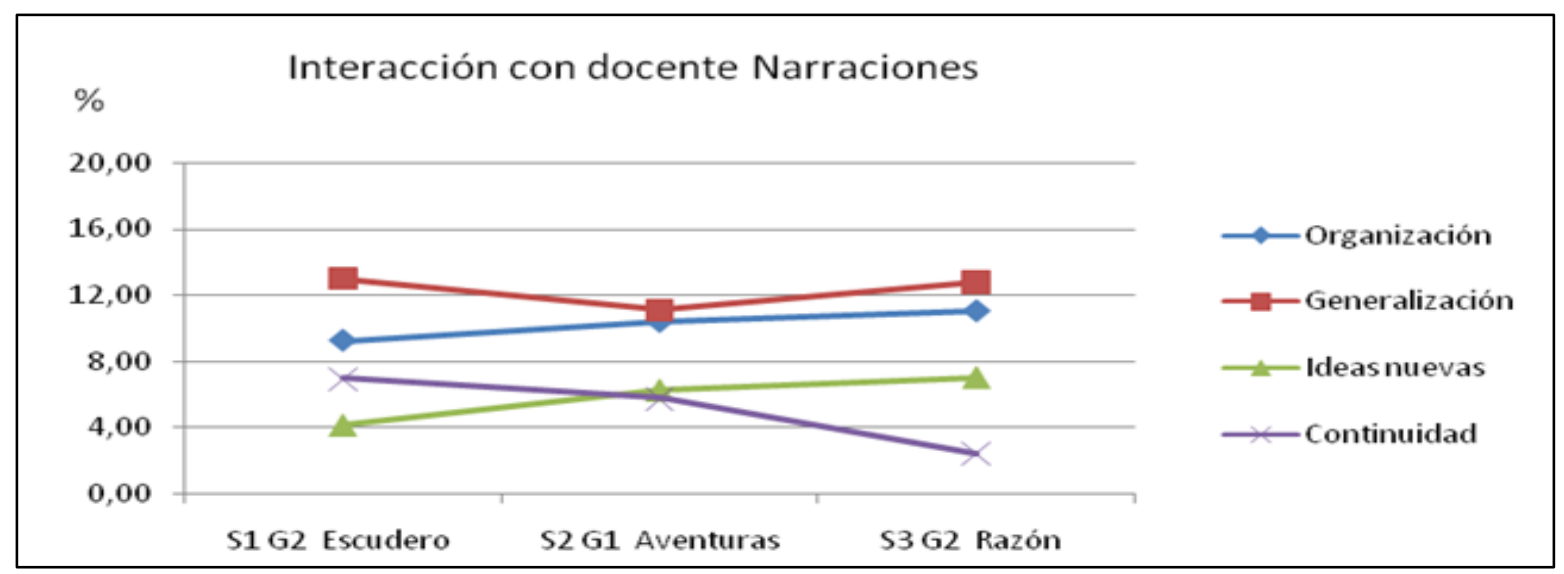

Figura 10. Soporte comunicativo del taller: situaciones diferenciadas con presencia de la profesora.

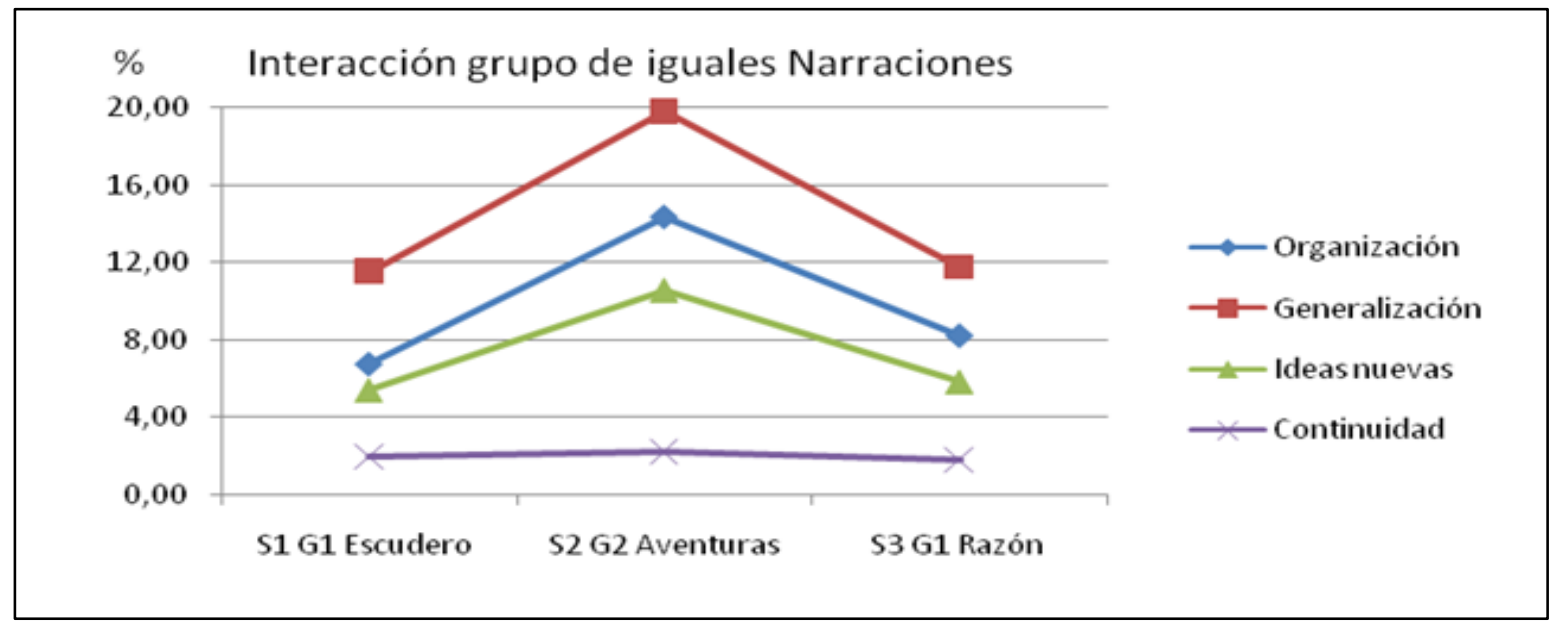

Figura 11. Soporte comunicativo del taller: situaciones diferenciadas sin presencia de la profesora.

Lo mismo ocurre en la gráfica correspondiente a la "Interacción grupo de iguales Narraciones", comprobamos que el grupo de iguales es capaz de controlar las rutinas escolares que hacen que funcione el grupo, gestionando y activando la participación conjunta. Los patrones de actividad del grupo de iguales son similares a la situación en la que está presente la profesora, con porcentajes de utilización de estrategias de organización y generalización entre el $12 \%$ y el 8 . En la segunda sesión (S2 G2 Aventuras) se eleva el porcentaje de estrategias de generalización hasta un $20 \%$. Este resultado se interpreta en base al proceso narrativo de la sesión, en el que los miembros del grupo se centraron en contenidos cercanos y significativos para elaborar su texto, intensificando su participación. La negociación llevada a cabo por el grupo, ha quedado reflejada en las estrategias discursivas dirigidas a la reflexión de significados y en las estrategias de la organización de la propia tarea y de la interacción social.

De forma paralela, la utilización de estrategias de aportación de ideas nuevas se ha incrementado a la vez que las estrategias más representadas. En este caso podemos pensar que el manejo de contenidos significativos para los estudiantes puede favorecer la conexión de nuevas ideas. 


\section{Conclusiones}

En un intento de recapitulación e integración de la teoría y la práctica de este trabajo, destacamos algunos de los aspectos más relevantes en la doble articulación entre la interacción y el discurso, y entre el discurso y la apropiación del conocimiento, propia de un proceso mental construido por los participantes para contextualizar su práctica (Mercer, 2001; van Dijk 2011). En relación a los objetivos planteados, la práctica educativa que se ha implementado como taller de historias ha permitido aproximar los contenidos curriculares establecidos a la realidad e intereses de los estudiantes. Docente y estudiantes han compartido vivencias extraescolares y trabajo en el aula, dialogando y reflexionando sobre su contenido para reconstruir nuevos significados sobre el papel. En la reconstrucción del taller de historias, el análisis de la interacción social nos ha mostrado la relación que se establece entre el objeto de conocimiento, en este caso el lenguaje escrito, los alumnos y alumnas que trabajan con dicho objeto y los agentes educativos que actúan de mediadores. De forma inductiva hemos descrito los patrones discursivos que se repiten a lo largo de la actividad educativa y su funcionalidad. Las estrategias sociocognitivas más utilizadas en el taller de composición textual narrativa se relacionan con el trabajo de construcción conjunta de significados, denominadas estrategias de generalización y con la organización social del grupo de trabajo y el procedimiento de cada tarea, denominadas estrategias de organización de la actividad conjunta. Es decir, los procedimientos de reflexión metacognitiva (Álvarez Angulo, 2006; Camps y Uribe, 2008) y la planificación y organización de las tareas han encaminado la comprensión y reconstrucción de las historias.

El análisis de las dos situaciones de trabajo, nos muestra como agentes educativos tanto a la profesora como al grupo de iguales. El papel de guía en el proceso de composición textual se ha relacionado con las exigencias de la tarea que han marcado la utilización de unas u otras estrategias sociocognitivas por parte de los participantes. Cabe destacar como guía del proceso de andamiaje del progreso sociocognitivo a la profesora, cuando maneja las rutinas de trabajo y realiza una progresiva cesión de traspaso de control a los estudiantes.

De acuerdo con Mercer y sus colaboradores (Warwick et. al., 2010), el rol de la docente "parece estar en la mente de los alumnos" lo que les permite captar y adaptar sus estrategias, reglas y procedimientos a su propio proceso de construcción. En definitiva, los participantes del taller se han convertido en protagonistas, implicados en el control y progreso de su aprendizaje.

\section{Referencias}

Romero, J. \& Luis, A. (2006). Reestructuración de los sistemas educativos y cambios en la formacion inicial del profesorado. Algunas reflexiones a partir del caso inglés. En J. M. Escudero \& A. Luis (Eds.), La formación del profesorado y la mejora de la educación. Políticas y prácticas (pp. 83-122). Barcelona, España: Octaedro. Álvarez Angulo, T. (2006). Didáctica de la escritura en la formación del profesorado. Lenguaje y textos, 23-24, 47-63.

Bakhtin, M. M. (1981). Discourse in the novel. En M. Holquist (Ed.), The dialogic imagination (pp. 259-422). Austin, TX: University of Texas Press.

Barton, D., \& Papen, U. (Eds.). (2010). The anthropology of writing: Understanding textually mediated worlds. New York: Continuum.

Bereiter, C., \& Scardamalia, M. (1987). The psychology of written composition. Hillsdale, N.J: Lawrence Erlbaum Associates.

Camps, A., y Uribe, P. (2008). La construcción del discurso escrito en un entorno académico: una visión de la dinámica del aprendizaje de los géneros discursivos. En La-Muralla (Ed.), El 
proceso de enseñar lenguas: investigaciones en didáctica de la lengua (Vol. 1, pp. 27-56). Madrid: La Muralla.

Coll, C. \& Onrubia, J. (1996). La construcción de significados compartidos en el aula: Actividad conjunta y dispositivos semióticos en el control y seguimiento mutuo entre profesor y alumnos. En C. Coll \& D. Edwards (Eds.), Enseñanza, aprendizaje y discurso en el aula (pp. 52 - 73). Madrid: Fundación Infancia y Aprendizaje.

Cubero, R., Cubero, M. \& Santamaría, A. (2008). La educación a través del discurso. Prácticas educativas y construcción discursiva del conocimiento en el aula. Revista de Educación, 346, 71-104.

Denzin, N. (2010). Moments, Mixed Methods, and Paradigm Dialogs. doi: 10.1177/1077800410364608. Recuperado de http://qix.sagepub.com/content/16/6/419.

Dörney, Z. (2007). Research methods in Applied Linguistics. Oxford University Press.

Ferreiro, E., Teberosky, A., \& Castorina, J. (2004). Sistemas de Escritura, Constructivismo y Educación. Buenos Aires: Homo Sapiens.

Gee, J. P. (1999). An introduction to discourse analysis. Theory and method. London y New York: Routledge.

Gee, J. P. (2008). Social linguistics and literacies: ideology in discourses. USA \& Canada: Routledge.

Hammersley, M., \& Atkinson, P. (2007). Ethnography. Principles in Practice. New York: Routledge

Hühn, P., Pier, J., Schmid, W., \& Schönert, J. (Eds.). (2009). HandbooK of Narratology. Berlin

Izquierdo-Magaldi, B. (2011) Estrategias discursivas de interacción en el aula. Construcción de textos expositivos y narrativos en grupos colaborativos. Tesis Doctoral Universidad de Alcalá.

Lacasa, P., \& GIPI. (2006). Aprendiendo periodismo digital. Historias de pequeñas escritoras. Madrid: Visor-Antonio Machado.

Lacasa, P., \& Reina, A. (2004). La televisión y el periódico en la escuela primaria: Imágenes, palabras e ideas. Tercer premio de investigación educativa 2002 (No. Proyecto financiado por el CIDE - MEC (BOE, 10-X-1997). Madrid: CIDE - MECD.

Mercer, N. (2001). Words and Minds. London: Routledge.

Mercer, N. \& Hodgkinson, S. (Eds.). (2008). Exploring talk in school. London: Sage

Olson, D. R., \& Torrance, N. (1991). Literacy as metalinguistics. En D. Olson (Ed.), Literacy and oraly (pp. 251-270). Cambridge: Cambridge University Press.

Olson, D. R., \& Torrance, N. (Eds.). (2009). The Cambridge Handbook of Literacy. University of Toronto

Rogoff, B. (1994). Developing understanding of the idea of communities of learners. Mind, Culture and Activity, 1(4), 209 - 229.

Rogoff, B. (2008). Observing Sociocultural Activity on three planes: Participatory Appropriation, Guided, Participation and Apprenticeship. En K. Hall, P. F. Murphy \& J. Soler (Eds.), Pedagogy and practice: culture and identities (pp 58-78). London: Routledge

Rogoff, B. (2012). Learning without lessons: Opportunities to expand knowledge. Infancia y Aprendizaje, 35 (2), 233-252.

Saló, N. (2006). Estrategias de Comunicación en el aula. Barcelona: Ceac.

Spindler, G. D., \& Hammond, L. A. (2006). Innovations in educational ethnography : theory, methods, and results. Mahwah, New Jersey: Lawrence Erlbaum Associates.

Street, B. (2003). What's "new" in New Literacy Studies? Critical approaches to literacy in theory and practice. Current issues in comparative education.columbia.edu, 5(2), 1-14.

Van Dijk, T. A. (2011). Discourse and Knowledge. En Paul Gee \& Michael (Eds.), Handbook of Discourse Analysis (pp.587-630). London: Routledge. 
Warwick, P., Mercer, N., Kershner, R., \& Staarman, J.K. (2010). In the mind and in the technology: The vicarious presence of the teacher in pupil's learning of science in collaborative group activity at the interactive whiteboard. Computers \& Education, 55(1), 350-36

\section{Sobre la autora}

Autora: Belén Izquierdo Magaldi

Institución: Universidad de Cantabria

E-mail: izquierdomb@unican.es

Información biográfica: Doctora en Psicología por la Universidad de Alcalá de Henares, en 2011. Licenciada en Psicología por la Universidad de Oviedo en 1985. Especialidad en

Psicología Pedagógica por la Universidad Complutense de Madrid, en 1986.

En la actualidad, Docente e Investigadora en el Área de Psicología Evolutiva y de la Educación del Departamento de Educación de la Universidad de Cantabria. Centrada en los procesos de composición textual en situaciones de interacción educativa, relacionados con una alfabetización en transformación.

ORCID: no disponible

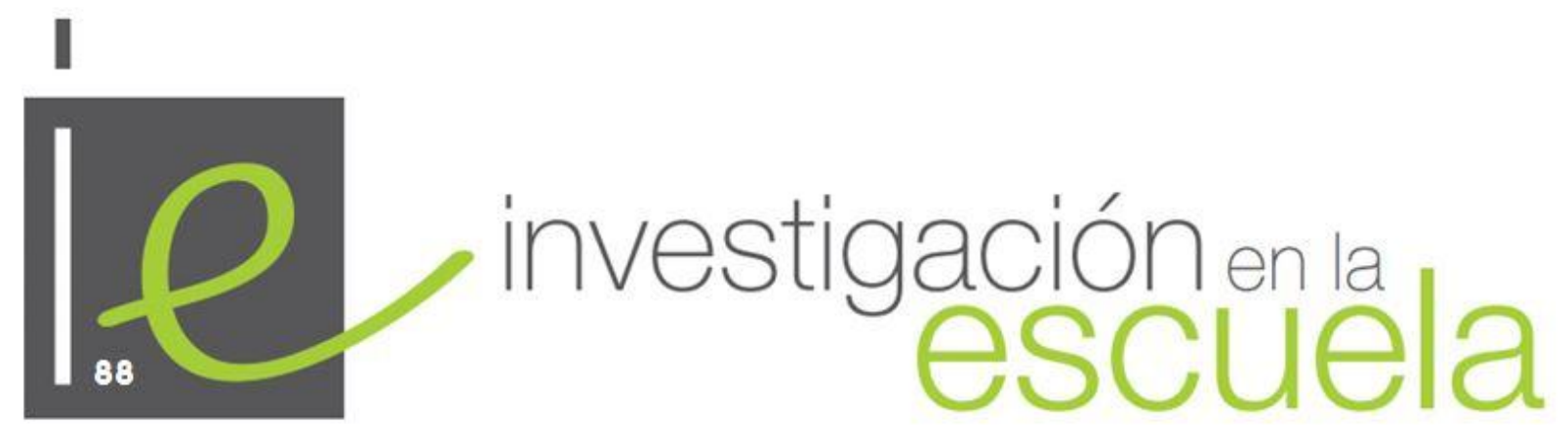

Revista académica evaluada por pares y de acceso abierto

Número 89

15 de noviembre de 2016

ISSN 2443-9991

(a)

SOMERIIGHISRESEEVED LOs/as lectores/as pueden copiar, mostrar, y distribuir este artículo, siempre y cuando se de crédito y atribución al autor/es y a Investigación en la Escuela, se distribuya con propósitos no-comerciales, no se altere o transforme el trabajo original. Más detalles de la licencia de Creative Commons se encuentran en http://creativecommons.org/licenses/by-nc-sa/3.0 Cualquier otro uso debe ser aprobado en conjunto por el autor/es, o Investigación en la Escuela.

Contribuya con comentarios y sugerencias en la web de la revista. Por errores y sugerencias contacte a investigacionescuela@,ddcc.uhu.es 


\title{
Investigación en la escuela
}

Consejo de dirección: Ana Rivero García (Universidad de Sevilla), Nicolás de Alba Fernández (Universidad de Sevilla), Pedro Cañal de León (Universidad de Sevilla), Francisco F. García Pérez (Universidad de Sevilla), Gabriel Travé González, (Universidad de Huelva), Francisco F. Pozuelos Estrada (Universidad de Huelva)

\author{
Dirección: Ana Rivero García y Nicolás de Alba Fernández \\ Técnico de edición: Francisco Javier López Sánchez
}

\section{Consejo editorial}

José Félix Angulo Rasco. Universidad de Cádiz Rosa Ma Ávila Ruiz. Universidad de Sevilla Pilar Azcárate Goded. Universidad de Cádiz Juan Bautista Martínez Rodríguez. Universidad de Granada

Nieves Blanco García. Universidad de Málaga Fernando Barragán Medero. Universidad de La Laguna José Carrillo Yáñez. Universidad de Huelva José Contreras Domingo. Universidad de Barcelona. Luis C. Contreras González. Universidad de Huelva Ana $\mathbf{M}^{\mathbf{a}}$ Criado García-Legaz. Universidad de Sevilla Rosario Cubero Pérez. Universidad de Sevilla José $\mathbf{M}^{\mathbf{a}}$ Cuenca López. Universidad de Huelva Jesús Estepa Giménez. Universidad de Huelva Rafael Feito Alonso. Universidad Complutense (Madrid)

Francisco José García Gallardo. Universidad de Huelva

Soledad García Gómez. Universidad de Sevilla J. Eduardo García Díaz. Universidad de Sevilla
Fernando Hernández Hernández. Universidad de Barcelona

Salvador Llinares Ciscar. Universidad de Alicante Alfonso Luque Lozano. Universidad de Sevilla Rosa Martín del Pozo. Universidad Complutense (Madrid)

José Martín Toscano. IES Fernando Herrera (Sevilla) Jaume Martínez Bonafé. Universidad de Valencia F. Javier Merchán Iglesias. Universidad de Sevilla Emilia Moreno Sánchez. Universidad de Huelva. Rosario Ortega Ruiz. Universidad de Córdoba Antonio de Pro Bueno. Universidad de Murcia Fco. de Paula Rodríguez Miranda. Universidad de Huelva

Pedro Sáenz-López Buñuel. Universidad de Huelva Antoni Santisteban Fernández. Universidad Autónoma (Barcelona)

Emilio Solís Ramírez. Catedrático de IES.

$\mathbf{M}^{\mathbf{a}}$ Victoria Sánchez García. Universidad de Sevilla. Magdalena Suárez Ortega. Universidad de Sevilla

\section{Consejo asesor}

Manuel Area Moreira. Universidad de La Laguna

Jaume Carbonell. Director Cuadernos de Pedagogía. Barcelona

César Coll. Universidad de Barcelona

Christopher Day. Universidad de Nothingham. U.K.

Juan Delval. Universidad Nacional de Educación a Distancia

John Elliott. Universidad de East Anglia. Norwich. U.K.

José Gimeno Sacritán. Universidad de Valencia

André Giordan. Universidad de Paris VII y Ginebra

Francisco Imbernón. Universidad de Barcelona

Ángel Pérez Gómez. Universidad de Málaga

Rafael Porlán Ariza. Universidad de Sevilla

Francesco Tonucci. Instituto de Pedagogía del C.N.R. Roma

Jurjo Torres Santomé. Universidad de A Coruña 
